\title{
POSITIVE QUATERNIONIC KÄHLER MANIFOLDS AND SYMMETRY RANK: II
}

\author{
FUQUAN FANG
}

\begin{abstract}
Let $M$ be a positive quaternionic Kähler manifold of dimension $4 m$. If the isometry group $\operatorname{Isom}(M)$ has rank at least $\frac{m}{2}+3$, then $M$ is isometric to $\mathbb{H} P^{m}$ or $G r_{2}\left(\mathbb{C}^{m+2}\right)$. The lower bound for the rank is optimal if $m$ is even.
\end{abstract}

\section{Introduction}

A quaternionic Kähler manifold $M$ is an oriented Riemannian $4 n$-manifold, $n \geq 2$, whose holonomy group is contained in $S p(n) S p(1) \subset S O(4 n)$. If $n=1$ we add the condition that $M$ is Einstein and self-dual. Equivalently, there exists a 3 -dimensional subbundle $S$ of the endmorphism bundle, $\operatorname{End}(T M, T M)$, locally generated by three anti-commuting almost complex structures $I, J, K=I J$ so that the Levi-Civita connection preserves $S$. It is well-known [3] that a quaternionic Kähler manifold $M$ is always Einstein, and is necessarily locally hyperkähler if its Ricci tensor vanishes. A quaternionic Kähler manifold $M$ is called positive if it has positive scalar curvature. By [13] (for $n=1$ ) and [20] (for $n \geq 2$, compare [16] [17]) a positive quaternionic Kähler manifold $M$ has a twistor space a complex Fano manifold. Hitchin [13] proved a positive quaternionic Kähler 4-manifold $M$ must be isometric to $\mathbb{C} P^{2}$ or $S^{4}$. Hitchin's work was extended by Poon-Salamon [19] to dimension 8, which proves that a positive quaternionic Kähler 8-manifold $M$ must be isometric to $\mathbb{H} P^{2}, G r_{2}\left(\mathbb{C}^{4}\right)$ or $G_{2} / S O(4)$.

This leads to the Salamon-Lebrun conjecture:

Every positive quaternionic Kähler manifold is a quaternionic symmetric space.

Very recently, the conjecture was further verified for $n=3$ in [12], using the approach initiated in [20] [19] (compare [17]). For a positive quaternionic Kähler manifold $M$, Salamon [20] proved that the dimension of its isometry group is equal to the index of certain twisted Dirac operator, by the Atiyah-Singer index theorem, which is a characteristic number of $M$ coupled with the Kraines 4 -form $\Omega$ (in analog with the Kähler form), and it was applied to prove that the isometry group of $M$ is large in lower dimensions (up to dimension 16).

By [17] a positive quaternionic Kähler $4 n$-manifold $M$ is simply connected and the second homotopy group $\pi_{2}(M)$ is a finite group or $\mathbb{Z}$, and $M$ is isometric to $\mathbb{H} P^{n}$ or $G r_{2}\left(\mathbb{C}^{n+2}\right)$ according to $\pi_{2}(M)=0$ or $\mathbb{Z}$.

An interesting question is to study positive quaternionic Kähler manifold in terms of its isometry group. This approach dates back to the work [19] for $n=2$ [12] for

Received by the editors January 31, 2004. Revision received August 23, 2007.

Supported by NSF Grant of China \#10671097 and the Capital Normal University. 
$n=3$ to proving the action is transitive, and [5] [18] for cohomogeneity one actions (and hence the isometry group must be very large). [4] classified positive quaternionic Kähler $4 n$-manifolds with isometry rank $n+1$, using an approach on hyper-Kähler quantizations. [6] establishes a connectedness theorem and using this tool the author proved that, a positive quaternionic Kähler $4 n$-manifolds of symmetry rank $\geq n-2$ must be either isometric to $\mathbb{H} P^{n}$ or $G r_{2}\left(\mathbb{C}^{n+2}\right)$, if $n \geq 10$.

In this paper we will combine Morse theory of the momentum map on quaternionic Kähler manifold [2] and the connectedness theorem in [6] to prove the following

Theorem 1.1. Let $M$ be a positive quaternionic Kähler manifold of dimension $4 m$. Then the isometry group Isom $(M)$ has rank (denoted by rank $(M))$ at most $(m+1)$, and $M$ is isometric to $\mathbb{H} P^{m}$ or $G r_{2}\left(\mathbb{C}^{m+2}\right)$ if $\operatorname{rank}(M) \geq \frac{m}{2}+3$.

Notice that the fixed point set of an isometric circle action on a quaternionic Kähler manifold of dimension $4 m$ is either a quaternionic Kähler submanifold or a Kähler manifold. In the latter case the fixed point set has dimension at most $2 m$ (the middle dimension of the manifold). Moreover, if a fixed point component is contained in $\mu^{-1}(0)$ then it must be a quaternionic Kähler submanifold, and if it is in the complement $M-\mu^{-1}(0)$ then it is Kähler (see [2]).

Theorem 1.2. Let $M$ be a positive quaternionic Kähler manifold of dimension $4 m$ with an isometric $S^{1}$-action. Assume $m \geq 3$. If $N$ is a fixed point component of codimension 4 , then $M$ is isometric to $\mathbb{H} P^{m}$ or $G r_{2}\left(\mathbb{C}^{m+2}\right)$.

The idea of proving Theorem 1.2 is as follows: by the assumption we know that the fixed point component $N \subset \mu^{-1}(0)$ since $4 m-4 \geq 2 m+1$ (cf. [2] Remark 3.2). Furthermore, by [8] the reduction $\mu^{-1}(0) / S^{1}$ has dimension at most $4 m-4$ (cf. [5]). We will prove in section 3 that $N=\mu^{-1}(0)$. This together with the equivariant Morse equality implies that $M=\mathbb{H} P^{m}$ if $b_{2}(M)=0$ (cf. Lemma 4.1) and so Theorem 1.2 follows by [17].

With Theorem 1.2 in hand, the proof of Theorem 1.1 follows by induction on $m$ and Theorems 2.1 and 2.4 .

Theorem 1.1 is optimal if $m$ is even since the rank of $\widetilde{G r}_{4}\left(\mathbb{R}^{m+4}\right)$ is $\frac{m}{2}+2$. We conjecture that when $m$ is odd, the lower bound for the rank in Theorem 1.1 may be improved by 1 , that is

Conjecture 1.3. Let $M$ be a positive quaternionic Kähler manifold of dimension $8 m+4$. Then $M$ is isometric to $\mathbb{H} P^{2 m+1}$ or $G r_{2}\left(\mathbb{C}^{2 m+3}\right)$ if $\operatorname{rank}(M) \geq m+3$.

\section{Acknowledgements}

The author is very grateful to F. Battagalia for many invaluable discussions through emails, who pointed out a gap in a previous version. Thanks should also go to the referee for correcting a number of typesetting errors. 


\section{Preliminaries}

In this section we recall some results on quaternionic Kähler manifolds needed in later sections.

Let $(M, g)$ be a quaternionic Kähler manifold of dimension $4 m$. Let $F \rightarrow M$ be the principal $S p(m) S p(1)$-bundle over $M$. Locally, $F \rightarrow M$ can be lifted to a principal $S p(m) \times S p(1)$-bundle, i.e., the fiberwise double cover of $F$. Let $E, H$ be the locally defined bundles associated to the standard complex representation of $S p(m)$ and $S p(1)$ respectively. The complexified cotangent bundle $T^{*} M_{\mathbb{C}}$ is isomorphic to $E \otimes_{\mathbb{C}} H$. The adjoint representations of $S p(m)$ and $S p(1)$ give two bundles $S^{2} E$ and $S^{2} H$ over $M$, respectively. Given the inclusion of the holonomy algebra $s p(m) \oplus s p(1)$ into $s o(4 m)$, the bundle $S^{2} E \oplus S^{2} H$ can be regarded as a subbundle of the bundle of 2-forms $\Lambda^{2} T^{*} M_{\mathbb{C}}$. The bundle $S^{2} H$ has fiber the Lie algebra $s p(1)$ and the local basis $\{I, J, K\}$ cooresponding to $i, j, k \in s p(1)$ which are almost complex structures satisfying that $I J=-J I=K$.

The Kraines 4 -form, $\Omega$, associated to a quaternionic Kähler manifold $M$, is a nondegenerate closed form which is defined by

$$
\Omega=\omega_{1} \wedge \omega_{1}+\omega_{2} \wedge \omega_{2}+\omega_{3} \wedge \omega_{3}
$$

where $\omega_{1}, \omega_{2}$ and $\omega_{3}$ are the locally defined 2-forms associated to the almost complex structures $I, J$ and $K$. The form $\Omega$ is globally defined and non-degenerate, namely $\Omega^{m}$ is a constant non-zero multiple of the volume form. It is well-known that $\Omega$ is parallel if and only if $M$ has holonomy in $S p(m) S p(1)$, if $m \geq 2$. Moreover, by [22], $M$ has holonomy in $S p(m) S p(1)$ if and only if $\Omega$ is closed, provided $m \geq 3$.

A quaternionic Kähler manifold may not have a global almost complex structure, e.g., the quaternionic projective space $\mathbb{H} P^{m}$. If $I, J, K$ are integrable and covariantly constant with respect the metric, the holonomy group reduces to $S p(m)$, quaternionic Kähler manifold is hyperkähler. Wolf [23] classified quaternionic symmetric spaces of compact type, they are $\mathbb{H} P^{m}$, the complex Grassmannian $\mathrm{Gr}_{2}\left(\mathbb{C}^{m+2}\right)$, and the oriented real Grassmannian $\widetilde{G r}_{4}\left(\mathbb{R}^{m+4}\right)$, and exactly one quaternionic symmetric space for each compact simple Lie algebra, $G_{2} / S O(4), F_{4} / S p(3) S p(1), E_{6} / S U(6) S p(1)$, $E_{7} / \operatorname{Spin}(12) S p(1), E_{8} / E_{7} S p(1)$.

Theorem 2.1 ([17]). (i) (Fininteness) For any $m \in \mathbb{Z}_{+}$, there are, modulo isometries and rescalings, only finitely many positive quaternionic Kähler manifolds of dimension $4 m$.

(ii) (Strong rigidity) Let $(M, g)$ be a positive quaternionic Kähler manifold of dimension $4 m$. Then $M$ is simply connected and

$$
\begin{aligned}
& 0,(M, g)=\mathbb{H} P^{m} \\
& \pi_{2}(M)=\left\{\mathbb{Z},(M, g)=G r_{2}\left(\mathbb{C}^{m+2}\right)\right. \\
& \text { finite with 2-torsion, otherwise }
\end{aligned}
$$

A submanifold $N$ in a quaternionic Kähler manifold is called a quaternionic submanifold if the locally defined almost complex structures $I, J, K$ preserve the tangent bundle of $N$. 
Proposition 2.2 ([9]). Any quaternionic submanifold in a quaternionic Kähler manifold is totally geodesic and quaternionic Kählerian.

Theorem 2.3 ([6]). Let $M$ be a positive quaternionic Kähler manifold of dimension $4 m$. Assume $f=\left(f_{1}, f_{2}\right): N \rightarrow M \times M$, where $N=N_{1} \times N_{2}$ and $f_{i}: N_{i} \rightarrow M$ are quaternionic immersions of compact quaternionic Kähler manifolds of dimensions $4 n_{i}, i=1,2$. Let $\Delta$ be the diagonal of $M \times M$. Set $n=n_{1}+n_{2}$. Then:

(2.3.1) If $n \geq m$, then $f^{-1}(\Delta)$ is nonempty.

(2.3.2) If $n \geq m+1$, then $f^{-1}(\Delta)$ is connected.

(2.3.3) If $f$ is an embedding, then for $i \leq n-m$ there is a natural isomorphism, $\pi_{i}\left(N_{1}, N_{1} \cap N_{2}\right) \rightarrow \pi_{i}\left(M, N_{2}\right)$ and a surjection for $i=n-m+1$.

As a direct corollary of (2.3.3) we have

Theorem 2.4 ([6]). Let $M$ be a positive quaternionic Kähler manifold of dimension $4 m$. If $N \subset M$ is a quaternionic Kähler submanifold of dimension $4 n$, then the inclusion $N \rightarrow M$ is $(2 n-m+1)$-connected.

\section{Hyperkähler quotient and Quaternionic Kähler quotient}

\section{a. Hyperkähler quotient}

Let $M$ be a hyperkähler manifold having a metric $g$ and covariantly constant complex structures $I, J, K$ which behave algebraically like quaternions:

$$
I^{2}=J^{2}=K^{2}=-1 ; I J=-J I=K
$$

Let $G$ be a compact Lie group acting on $M$ by isometries preserving the structures $I, J, K$. The group $G$ preserves the three Kähler forms $\omega_{1}, \omega_{2}, \omega_{3}$ corresponding to the complex structures $I, J, K$, so we may define moment maps $\mu_{1}, \mu_{2}, \mu_{3}$, respectively. These may be written as a single map

$$
\mu: M \rightarrow \mathfrak{g}^{*} \otimes \mathbb{R}^{3}
$$

Let

$$
\mu_{+}=\mu_{2}+i \mu_{3}: M \rightarrow \mathfrak{g}^{*} \otimes \mathbb{C}
$$

where $\mathfrak{g}^{*}$ is the dual space of the Lie algebra of $G$.

By [14] $\mu_{+}$is holomorphic, and so $N=\mu_{+}^{-1}(0)$ is a complex subvariety of $M$, with respect to the complex structure $I$. By definition, $\mu^{-1}(0)=N \cap \mu_{1}^{-1}(0)$. The hyperkähler quotient is the quotient space $\mu^{-1}(0) / G$, denoted by $M / / G$. In particular, if $\mu^{-1}(0)$ is a manifold and the induced $G$-action is free, then the hyperkähler quotient $M / / G$ is also a hyperKähler manifold. More generally, Dancer-Swann [5] proved that the hyperkähler quotient $M / / G$ may be decomposed into the union of hyperkähler manifolds, according to the isotropy decomposition of the $G$-action on $M$. However, it is wide open if the decomposition of $M / / G$ is a stratified topological space, as in the sympletic quotient case [21]. 
In this section we will consider the structure of this decomposition in the special case that $G=S^{1}$ and the action is semi-free, i.e., free outside the fixed point set.

Let us start with the standard example of isometric $S^{1}$-action on quaternionic linear space $\mathbb{H}^{n}$ defined by

$$
\varphi_{t}(u)=e^{2 \pi i t} u ; \quad t \in[0,1)
$$

where $i$ is one of the quaternionic units. With global quaternionic coordinates $\left\{u^{\alpha}\right\}$, $\alpha=1, \cdots, n$, the standard flat metric on $\mathbb{H}^{n}$ may be written as:

$$
d s^{2}=\sum_{\alpha} d \bar{u}^{\alpha} \otimes d u^{\alpha}
$$

where $\bar{u}^{\alpha}$ is the quaternionic conjugate of $u^{\alpha}$.

The Killing vector field $X$ of the above action is $\mathbb{H}$-valued:

$$
X^{\alpha}(u)=i u^{\alpha}
$$

which is triholomorphic.

Consider the $\mathbb{H}$-valued 2-form

$$
\omega=\sum_{\alpha} d \bar{u}^{\alpha} \wedge d u^{\alpha}
$$

Observe that $\omega$ is purely imaginary since $\omega+\bar{\omega}=0$. Note that $\omega=\omega_{1} i+\omega_{2} j+\omega_{3} k$, where $\omega_{i}$ is as above.

It is easy to see that the moment map (cf. [7])

$$
\mu^{X}=\sum_{\alpha} \bar{u}^{\alpha} i u^{\alpha}: \mathbb{H}^{n} \rightarrow i \mathbb{R}^{3}
$$

Write $\mathbb{H}^{n}=\mathbb{C}^{n} \oplus j \mathbb{C}^{n}$. The zero set of the holomorphic moment map $\mu_{+}^{-1}(0)$ is the complex algebraic variety of complex dimension $(2 n-1)$ :

$$
\left\{(a, b) \in \mathbb{C}^{n} \oplus j \mathbb{C}^{n}:\langle a, \bar{b}\rangle=\sum_{\alpha} a^{\alpha} b^{\alpha}=0\right\}
$$

In particular, if $n=1$, then $\mu_{+}^{-1}(0)$ is a reducible algebraic curve with two irreducible components the standard complex lines.

The hyperkähler quotient $\mathbb{H}^{n} / / S^{1}$ is an open cone over a $(4 n-5)$-dimensional manifold $W$ :

$$
W=\left\{(a, b) \in \mathbb{C}^{n} \oplus \mathbb{C}^{n}:|a|^{2}=|b|^{2}=1,\langle a, \bar{b}\rangle=\sum_{\alpha} a^{\alpha} b^{\alpha}=0\right\} / S^{1}
$$

In particular, $\mathbb{H} / / S^{1}=\{0\}$, a single point.

Theorem 3.1. Let $M^{4 m}$ be a hyperkähler manifold with an isometric effective $S^{1}$ action preserving the hyperkähler structure. Let $\mu$ be the hyperkähler moment map. If $Y \subset M^{S^{1}} \cap \mu^{-1}(0)$ is a connected fixed point component of dimension $4 m-4$, then $Y \subset M / / S^{1}$ is a connected component.

Before we start the proof, let us give the analog of the classical Darboux-Weinstein theorem for complex symplectic manifolds. Recall that a complex symplectic manifold $W$ is a complex manifold with a complex value symplectic form $\omega^{c}=\omega_{2}+i \omega_{3}$, where $\omega_{2}, \omega_{3}$ are two real value symplectic forms. 
Lemma 3.2 (Relative equivariant Darboux theorem). Let $G$ be a compact Lie group acting on a complex symplectic manifold $W$. Let $\omega_{0}^{c}$ and $\omega_{1}^{c}$ be two $G$-equivariant complex symplectic forms on $W$. Assume they coincide on a closed $G$-invariant complex symplectic submanifold $V$. Then there exists a $G$-invariant neighborhood $U_{0}$ of $V$ in $W$ and a $G$-equivariant map

$$
\psi: U_{0} \rightarrow W
$$

such that

$$
\left.\psi\right|_{V}=I d_{V} \text { and } \psi^{*} \omega_{1}=\omega_{0}
$$

Proof. We apply the path method due to Moser. Consider the form $\omega_{t}^{c}=\omega_{0}^{c}+t\left(\omega_{1}^{c}-\right.$ $\left.\omega_{0}^{c}\right)$. This is a closed complex value 2 -form which is non-degenerate on $V$ and thus on a small $G$-invariant tubular neighborhood $U$ of $V$.

Since $\omega_{0}^{c}$ and $\omega_{1}^{c}$ are closed, and so is $\omega_{0}^{c}-\omega_{1}^{c}$, and thus we may find a complex value 1-form $\beta$ on a neighborhood of $V$ such that $d \beta=\omega_{0}^{c}-\omega_{1}^{c}$. Indeed, $U$ is $G$-equivariant diffeomorphic to an open neighborhood of the zero section of a $G$-equivariant vector bundle on $V$, hence retracts on $V$. By applying the Poincaré lemma to $\omega_{0}^{c}-\omega_{1}^{c}$ we get the $G$-invariant 1-form $\beta$, that can be chosen so that $\beta_{x}=0$ for all $x \in V$.

The complex value closed 2-form $\omega_{t}^{c}$ being non-degenerate, it defines a time dependent $G$-invariant vector field $X_{t}$ such that the contraction $i_{X_{t}} \omega_{t}^{c}=\beta$. Note that $X_{t}=0$ on $V$. Its flow $\varphi_{t}$ keeps $V$ fixed, and thus one can find a $G$-invariant neighborhood $U_{0}$ of $V$ where $\varphi_{t}$ is defined and such that $\varphi_{t}\left(U_{0}\right) \subset U$.

Therefore,

$$
\frac{d}{d t}\left[\varphi_{t}^{*} \omega_{t}^{c}\right]=\varphi_{t}^{*}\left[\frac{d \omega_{t}^{c}}{d t}+\mathfrak{L}_{X_{t}} \omega_{t}^{c}\right]=\varphi_{t}^{*}\left[\omega_{1}^{c}-\omega_{0}^{c}+\omega_{0}^{c}-\omega_{1}^{c}\right]=0
$$

because $\mathfrak{L}_{X_{t}} \omega_{t}^{c}=d i_{X_{t}} \omega_{t}^{c}+i_{X_{t}} d \omega_{t}^{c}=d \beta$ using the Cartan formula and the definition of $\varphi_{t}$. The form $\varphi_{t}^{*} \omega_{t}^{c}$ does not depend on $t$, and it equals $\omega_{0}^{c}$ for $t=0$. Put $\psi=\varphi_{1}$ the desired result follows.

Now we are ready to prove

Proof of Theorem 3.1. Recall that $\omega^{c}=\omega_{2}+i \omega_{3}$ defines a complex symplectic structure on $M$. Choose an open ball $V$ in $Y$ with restricted complex symplectic structure. The complex normal bundle of $V$ in $M$ is a trivial complex vector bundle $V \times \mathbb{C}^{2}$. The $S^{1}$-action on the bundle is the product action of a trivial action on $V$ and an effective complex linear action on $\mathbb{C}^{2}$, saying, $t \cdot\left(z_{1}, z_{2}\right)=\left(t^{p} z_{1}, t^{q} z_{2}\right)$ for some $p, q \in \mathbb{Z}$. Since the hyperkähler metric is $S^{1}$-invariant, the normal exponential map $\varphi=\exp _{V}: V \times \mathbb{C}^{2} \rightarrow M$ defines an $S^{1}$-equivariant diffeomorphism from an $S^{1}$-invariant tubular neighborhood $V_{0}$ of $V \times\{0\}$ in $V \times \mathbb{C}^{2}$ to an $S^{1}$-invariant tubular neighborhood $U_{0}$ of $V$ in $M$. Note that $p q=-1$ since the action is effective and preserves the hyperkähler structure.

Consider the $S^{1}$-invariant complex symplectic form $\omega_{0}^{c}=\left.\omega^{c}\right|_{V} \times\left(d z_{1} \wedge d z_{2}\right)$ on $V \times$ $\mathbb{C}^{2}$. The pullback form $\left(\varphi^{-1}\right)^{*} \omega_{0}^{c}$ and $\left.\omega^{c}\right|_{U_{0}}$ are both $S^{1}$-invariant complex symplectic forms on $U_{0}$ which coincide on $V$. By Lemma 3.2 there exists an $S^{1}$-equivariant diffeomorphism $\psi$ such that $\left.\psi^{*} \omega^{c}\right|_{U_{0}}=\left(\varphi^{-1}\right)^{*} \omega_{0}^{c}$. Therefore, $\left.(\psi \circ \varphi)^{*} \omega^{c}\right|_{U_{0}}=\omega_{0}^{c}$.

The moment map $\left.\mu_{+}\right|_{U_{0}}$ of $\left(U_{0}, \omega^{c}\right)$ with the restricted $S^{1}$-action may be identified with the moment map of $\left(V_{0}, \omega_{0}^{c}\right)$ with the product action. Thus,

$$
\mu_{+}^{-1}(0) \cap U_{0} \cong(V \times\{0\}) \times L \cap V_{0}
$$


where $L \subset \mathbb{C}^{2}$ is the zero locus of the moment map of $\left(\mathbb{C}^{2}, d z_{1} \wedge d z_{2}\right)$ with the above mentioned $S^{1}$-action. By the paragraph before Theorem 3.1 we already knew that $L$ is the reducible curve given by $z_{1} z_{2}=0$. Therefore, $\mu_{+}^{-1}(0) \cap U_{0}$ is the union of two $S^{1}$-invariant complex submanifolds, $N_{1}, N_{2} \subset M$ (with respect to the complex structure $I$ ), and the $S^{1}$-action on $N_{1}$ (resp. $N_{2}$ ) has a real codimension 2 fixed point set (e.g., $\left.N_{1} \cong(V \times\{0\}) \times\left\{\left(z_{1}, 0\right): z_{1} \in \mathbb{C}\right\} \cap V_{0}\right)$. Obviously, both $N_{1}$ and $N_{2}$ have induced Kähler structures (w.r.t. $I$ ), and the restriction of $\mu_{1}$ on $N_{1}$ (resp. $N_{2}$ ) equals the moment map of the restricted $S^{1}$-action on $N_{1}$ (resp. $N_{2}$ ) with the induced Kähler structure.

By definition, $\mu^{-1}(0) \cap U_{0}=\mu_{+}^{-1}(0) \cap U_{0} \cap \mu_{1}^{-1}(0)=\left(\left.\mu_{1}\right|_{N_{1}}\right)^{-1}(0) \cup\left(\left.\mu_{1}\right|_{N_{2}}\right)^{-1}(0)$. Consider the moment map $\mu_{1}$ of the Kähler manifold $\left(N_{1}, \omega_{1}\right)$ with the restricted $S^{1}$-action. In a small tubular neighborhood $W_{1}$ of the fixed point component $V \subset$ $N_{1}$ of codimension 2, by [10] $\left(\left.\mu_{1}\right|_{N_{1}}\right)^{-1}(0)$ is a conic bundle over the fixed point set $V$. Since $\left(\left.\mu_{1}\right|_{N_{1}}\right)^{-1}(0)$ has dimension $4 m-4$, where $\operatorname{dim}\left(N_{1}\right)=4 m-2$, thus, $\left(\left.\mu_{1}\right|_{N_{1}}\right)^{-1}(0) \cap W_{1}=V$. Similarly, $\left(\left.\mu_{1}\right|_{N_{2}}\right)^{-1}(0) \cap W_{2}=V$, where $W_{2}$ is a small tubular neighborhood of $V$ in $N_{2}$. Therefore, $\mu^{-1}(0) \cap U_{0}^{\prime}=V$ for a possibly smaller tubular neighborhood $U_{0}^{\prime}$ of $V$ in $M$. By definition of $M / / S^{1}$ we conclude the desired result.

\section{b. Quaternionic Kähler quotient}

Let $M$ be a quaternionic Kähler manifold with non-zero scalar curvature. If $G$ acts on $M$ by isometries, there is a well-defined moment map, which is a section $\mu \in \Gamma\left(S^{2} H \otimes \mathfrak{g}^{*}\right)$ solving the equation

$$
\langle\nabla \mu, X\rangle=\sum_{i=1}^{3} \overline{I_{i} X} \otimes I_{i}
$$

for each $X \in \mathfrak{g}$; where $\bar{X}=g(X, \cdot)$ denote the 1 -form dual to $X$ with respect to the Riemannian metric. Equivalently, the above equation may be written in the following form similar to the symplectic case

$$
d \mu(X)=i_{X} \Omega
$$

A nontrivial feature for quaternionic quotient is, the section $\mu$ is uniquely determined if the scalar curvature is nonzero. Moreover, only the preimage of the zero section of the moment map, $\mu^{-1}(0)$, is well-defined.

Theorem 3.3 ([8]). Let $M^{4 n}$ be a quaternionic Kähler manifold with nonzero scalar curvature acted on isometrically by $S^{1}$. If $S^{1}$ acts freely on $\mu^{-1}(0)$ then $\mu^{-1}(0) / S^{1}$ is a quaternionic Kähler manifold of dimension $4(n-1)$.

Since the proof of the Galicki-Lawson's theorem is local, so if the circle action is free on a piece of the manifold, the same result applies to the moment map on this piece.

Let $f=\|\mu\|^{2}$. By [2] the critical set of $f$ is the union of the zero set $f^{-1}(0)=\mu^{-1}(0)$ and the fixed point set of the circle action. Moreover, the zero set $\mu^{-1}(0)$ is connected, and a fixed point component is either contained in $\mu^{-1}(0)$ or does not interesect with 
$\mu^{-1}(0)$. Following [2] the Morse function $f$ is called equivariantly perfect over $\mathbb{Q}$ if the equivariant Morse equialities hold, that is if

$$
\hat{P}_{t}(M)=\hat{P}_{t}\left(\mu^{-1}(0)\right)+\sum t^{\lambda_{F}} \hat{P}_{t}(F)
$$

where the sum ranges over the set of connected components outside $\mu^{-1}(0)$ of the fixed point set, $\lambda_{F}$ is the index of $F$, and $\hat{P}_{t}$ is the equivariant Poincaré polynomial for the equivariant cohomology with coefficients in $\mathbb{Q}$.

Theorem 3.4 ([2]). Let $M^{4 n}$ be a quaternionic Kähler manifold acted on isometrically by $S^{1}$. Then the Morse function $\|\mu\|^{2}$ is equivariantly perfect over $\mathbb{Q}$.

Proposition $3.5([2])$. Let $M^{4 n}$ be a positive quaternionic Kähler manifold acted on isometrically by $S^{1}$. Then every connected component of the fixed point set, not contained in $\mu^{-1}(0)$, is a Kähler submanifold of $M-\mu^{-1}(0)$ of real dimension less than or equal to $2 n$ whose Morse index is at least $2 n$, with respect to the function $f$.

For each quaternionic Kähler manifold $M$ with non-zero scalar curvature, following [22], let $\mathfrak{u}(M)$ denote the $H^{*} /\{ \pm 1\}$-bundle over $M$ :

$$
\mathfrak{u}(M)=F \times_{S p(n) \operatorname{Sp}(1)}\left(H^{*} /\{ \pm 1\}\right)
$$

where $F$ is the principal $S p(n) S p(1)$-bundle over $M$. Let $\pi: \mathfrak{u}(M) \rightarrow M$ denote the bundle projection. Obviously, if $G$ is acts on $M$ by isometries, $G$ can be lifted to a $G$-action on $\mathfrak{u}(M)$. It is proved in [22] that, if the scalar curvature is positive, $\mathfrak{u}(M)$ has a hyperkähler structure which is preserved by the lifted $G$-action. Let $\hat{\mu}$ denote the moment map of the lifted $G$-action on $\mathfrak{u}(M)$. By [22] Lemma $4.4 \hat{\mu}=\mu \circ \pi$.

Lemma 3.6. Let $M$ be a positive quaternionic Kähler manifold of dimension $4 n$. Assume that $S^{1}$ acts on $M$ effectively by isometries. Let $\mu \in \Gamma\left(S^{2} H\right)$ be its moment map. If $N \subset \mu^{-1}(0)$ is a fixed point component of codimension 4 , then $N=\mu^{-1}(0)$.

Proof. Let $\mathfrak{u}(M)$ be as above. By Proposition 4.2 of [5], at the fixed point $x \in N$, the isotropy representation of $S^{1}$ in $S O(3) \cong \operatorname{Aut}\left(\mathfrak{u}(M)_{x}\right)$ factors through a finite group and hence the representation is trivial, where $\operatorname{Aut}\left(\mathfrak{u}(M)_{x}\right)$ is the isomorphism group of the fiber at $x$ preserving the quaternionic structure, and $\mathfrak{u}(M)_{x}=\pi^{-1}(x)$ is the fiber of the bundle at $x$. Therefore, $\pi^{-1}(N)$ is also a fixed point component of the lifted $S^{1}$-action on $\mathfrak{u}(M)$ of codimension 4 .

By [22] Lemma 4.4 we see that $\pi^{-1}(N) \subset \hat{\mu}^{-1}(0)$, where $\hat{\mu}$ is the moment map for the lifted $S^{1}$-action on $\mathfrak{u}(M)$. Now $S^{1}$ acts on the normal slice of $\pi^{-1}(N)$ in $\mathfrak{u}(M)$ through a representation in $S p(1)$. For dimension reasoning, this representation is faithful, otherwise, a finite order subgroup of $S^{1}$ acts trivially on the whole manifold $\mathfrak{u}(M)$ and so on $M$, a contradiction to the effectiveness of the action from our assumption. Therefore, $S^{1}$ acts semi-freely on a neighborhood of $\pi^{-1}(N)$ in $\mathfrak{u}(M)$. By now we may apply Theorem 3.1 to show that $\pi^{-1}(N)$ is a connected component of $\hat{\mu}^{-1}(0)$. Since the moment map $\hat{\mu}$ projects to the moment map $\mu$, we conclude $N$ is also a connected component of $\mu^{-1}(0)$. By [2] $\mu^{-1}(0)$ is connected, thus $N=\mu^{-1}(0)$, the desired result follows. 


\section{Proof of Theorem 1.2}

Theorem 1.2 follows readily from the following Lemma and Theorem 2.1, where the dimension bound $m \geq 3$ implies that the fixed point component of codimension 4 has to be contained in $\mu^{-1}(0)$, by Proposition 3.5.

Lemma 4.1. Let $M$ be a positive quaternionic Kähler $4 n$-manifold with an isometric $S^{1}$-action where $m \geq 3$. Let $\mu$ be the moment map. Assuming $b_{2}(M)=0$. If $N \subset \mu^{-1}(0)$ is a fixed point component of dimension $4 m-4$ of the circle action, then $M$ is isometric to $\mathbb{H} P^{m}$.

Proof. By Lemma $3.5 \mu^{-1}(0)=N$, therefore $S^{1}$ acts trivially on $\mu^{-1}(0)$.

By Theorem 3.4

$$
\hat{P}_{t}(M)=\hat{P}_{t}(N)+\sum_{F} t^{\lambda_{F}} \hat{P}_{t}(F)
$$

where $F$ runs over fixed point components outside $N$, and $\lambda_{F}$ the Morse index of $F$. By Proposition 3.5 the Morse index $\lambda_{F} \geq 2 n$ and are all even numbers. Thus the inclusion $N \rightarrow M$ is a $(2 n-1)$-equivalence.

By [2] Lemma $2.2 \hat{P}_{t}(M)=P_{t}(M) P_{t}\left(B S^{1}\right)$. Since $S^{1}$ acts trivially on $F$ and $N$, we get that $\hat{P}_{t}(F)=P_{t}(F) P_{t}\left(B S^{1}\right)$ and $\hat{P}_{t}(N)=P_{t}(N) P_{t}\left(B S^{1}\right)$. The above identity reduces to

$$
P_{t}(M)-P_{t}(N)=\sum_{F} t^{\lambda_{F}} P_{t}(F)
$$

Observe that the last two terms of the left hand side, according to increasing degree in $t$, is $b_{2}(M) t^{4 m-2}+t^{4 m}=t^{4 m}$ by Poincaré duality.

If $F$ is a fixed point component outside $\mu^{-1}(0)$ such that $\operatorname{dim}_{\mathbb{R}} F>0$, we claim that $\operatorname{dim}_{\mathbb{R}} F+\lambda_{F} \leq 4 m-4$. Otherwise, by the above identity $\operatorname{dim}_{\mathbb{R}} F+\lambda_{F}=4 m-2$ is impossible, and if the even integer $\operatorname{dim}_{\mathbb{R}} F+\lambda_{F}=4 m$, we conclude that the coefficients of $t^{4 m-2}$ of the right hand side is also non-zero, since $F$ must be a compact Kähler manifold (by Proposition 3.4) and so $P_{t}(F)$ has nonzero coefficient at every even degree not larger than the dimension. A contradiction.

By the above, the identity also implies that there is no isolated fixed point outside $\mu^{-1}(0)$ with Morse index $4 m-2$, and there is an isolated fixed point with Morse index $4 m$.

Put all together, by Morse theory, up to homotopy equivalence,

$$
M \simeq N \cup_{i} e^{\lambda_{i}} \cup e^{4 m}
$$

where $2 m \leq \lambda_{i} \leq \operatorname{dim}_{\mathbb{R}} F+\lambda_{F} \leq 4 m-4$, and $e^{i}$ denotes cell of dimension $i$. Therefore $H^{4 m-2}(M, N)=0$. By duality $H_{2}(M-N) \cong H^{4 m-2}(M, N)=0$. Since the codimension of $N$ is 4 , it follows that $H_{2}(M) \cong H_{2}(M-N)=0$. Therefore by Theorem $2.1 M=\mathbb{H} P^{m}$. The desired result follows. 


\section{Proof of Theorem 1.1}

Let $M$ be a positive quaternionic Kähler manifold of dimension $4 m$. We call the rank of the isometry group $\operatorname{Isom}(M)$ the symmetry $\operatorname{rank}$ of $M$, denoted by $\operatorname{rank}(M)$. By [6] we know that $\operatorname{rank}(M) \leq m+1$.

Proof of Theorem 1.1. Let $r=\operatorname{rank}(M)$. Consider the isometric $T^{r}$-action on $M$. Note that the $T^{r}$-action on $M$ must have non-empty fixed point set since the Euler characteristic $\chi(M)>0$ by [20]. Consider the isotropy representation of $T^{r}$ at a fixed point $x \in M$, which must be a representation through the local linear holonomy $S p(m) S p(1)$ representation at $T_{x} M \cong \mathbb{H}^{m}$. If there is a stratum (a fixed point set of an isotropy group of rank $\geq 1$ ) of codimension 4 , then it must be contained in $\mu^{-1}(0)$ if $m \geq 3$ (by [2] or Proposition 3.6). By Theorem 2.1 and Lemma 4.1 the desired result follows. Thus we can assume that at $x$, the isotropy representation does not have any codimension 4 linear subspace fixed by some rank 1 subgroup of $T^{r}$. Let $N$ be a maximal dimensional submanifold of $M$ passing through $x$ fixed by a circle subgroup of $T^{r}$.

Case (i): If $m=0(\bmod 2)$.

By the above assumption $4 m-8 \geq \operatorname{dim} N \geq 2 m+4 \operatorname{since} \operatorname{rank}(N)=r-1 \geq \frac{m}{2}+2$, by Lemma 2.1 of [6]. Note that $N$ is a quaternionic Kähler manifold since $N \subset \mu^{-1}(0)$. By Theorem 2.4 we see that $\pi_{2}(N) \cong \pi_{2}(M)$. By Theorem 2.1 it suffices to prove $\pi_{2}(N)=0$ or $\mathbb{Z}$. By induction we may consider $T^{r}$-action on $N$, and applying Lemma 4.1 once again. Finally it suffices to consider the case where a 16-dimensional quaternionic Kähler submanifold of $M, M^{16}$, with an effective isometric action by torus of rank $\geq 5$. In this case there is a quaternionic Kähler submanifold $M^{12} \subset M^{16}$ fixed by a circle group (cf. [6]). By Lemma 4.1, Theorem 2.1 and Theorem 2.4 $M^{16}=\mathbb{H} P^{4}$ or $\mathrm{Gr}_{2}\left(\mathbb{C}^{6}\right)$, the desired result follows.

Case (ii): If $m=1(\bmod 2)$.

Similar to the above $\operatorname{dim} N \geq 2 m+6$ for the same reasoning. By Theorem 2.4 $\pi_{2}(N) \cong \pi_{2}(M)$. The same argument by induction reduces the problem to the case of a quaternionic Kähler submanifold of dimension $20, M^{20}$, with an effective isometric torus action of rank $\geq 6$. Once again the argument in [6] shows that $M^{20}$ has a quatertnionic submanifold $M^{16}$ of rank $\geq 5$. By (i) we see that $M^{16}=\mathbb{H} P^{4}$ or $\mathrm{Gr}_{2}\left(\mathbb{C}^{6}\right)$. By Theorem 2.1 and Theorem 2.4 again we complete the proof.

\section{References}

[1] M. Atiyah and I.M. Singer, The index of elliptic operators III, Ann. Math. 87 (1968), 546-604

[2] F. Battaglia, Circle actions and Morse theory on quaternion-Kähler manifolds, J. London Math. Soc. 59 (1997), 345-358

[3] M. Berger, Sur les groupes d'holonomie des varietes a connexion affine et des varietes riemanniennes, Bull. Soc. Math. France, 83 (1955), 279-330

[4] R. Bielawski, Compact hyperkähler $4 n$-manifolds with a local tri-Hamiltonian $\mathbb{R}^{n}$-action, Math. Ann. 314 (1999), 505-528

[5] A. Dancer and A. Swann, Quaternionic Kähler manifolds of cohomogeneity one, Internat. J. Math. 10 (1999)541-570

[6] F. Fang, Positive quaternionic Kähler manifold and symmetry rank, J. Rein. Angew. Math., 576 (2004), 149-165 
[7] K. Galicki, A generalization of the momentum mapping construction for quaternionic Kähler manifolds, Comm. Math. Phys. 108 (1987), 117-138

[8] K. Galicki and B. Lawson, Quaternionic reduction and quaternionic orbifolds, Math. Ann. 282 (1988), 1-21

[9] A. Gray, A note on manifolds whose holonomy is a subsgroup of $\operatorname{Sp}(n) \operatorname{Sp}(1)$ Mich. Math. J., 16 (1965), 125-128

[10] V. Guillemin, Moment maps and combinatorial invariants of Hamiltonian $T^{n}$-spaces, Progress in Mathematics,122, Birkhäuser, 1994

[11] V. Guillemin and S. Sternberg, A normal form for the moment map, Differential geometric methods in mathematical physics (Jerusalem, 1982), 161-175, Math. Phys. Stud., 6, Reidel, Dordrecht, 1984.

[12] H. Herrera and R. Herrera, Â-genus on non-spin manifolds with $S^{1}$ actions and the classification of positive quaternionic Kähler 12-manifolds, J. Diff. Geom., 61 (2002), 341-364

[13] N. Hitchin, Kähler twistor spaces, Proc. London Math. Soc., 43 (1981), 133-150

[14] N. Hitchin, A. Karlhede, U. LindstrÖm and M. Rocek, Hyperkähler metrics and supersymmetry, Comm. Math. Phys., 108 (1987), 535-589

[15] V. Kraines, Topology of quaternionic Kähler manifolds, Trans. Amer. Math. Soc., 122 (1966), 357-367

[16] C. Lebrun, Fano manifolds, contact structures and quaternionic geometry, Inter. J. Math.,6 (1995), 419-437

[17] C. Lebrun and S. Salamon, Strong rigidity of positive quaternionic Kähler manifolds, Invent. Math., 118 (1994), 109-132

[18] F. Podesta and L. Verdiani, A note on quaternionic-Kähler manifolds, Internat. J. Math., 11 (2000), 279-283

[19] Y.S. Poon and S. Salamon, Eight-dimensional quaternionic Kähler manifolds with positive scalar curvature, J. Diff. Geom., 33 (1991), 363-378

[20] S. Salamon, Quaternionic Kähler manifolds, Invent. Math.,67 (1982), 143-171

[21] R. Sjamaar and E. Lerman, Stratified symplectic spaces and reduction, Ann. Math., 134 (1991), $375-422$

[22] A. Swann, Singular moment maps and quaternionic geometry, Banach Center Publ. 39 (1997). 143-153

[23] J.A. Wolf, Complex homogeneous contact structures and quaternionic symmetric spaces, J. Math. Mech., 14 (1965), 1033-1047

Department of Mathematics, Capital Normal University, Beijing, P.R.China

E-mail address: fuquan_fang@yahoo.com 